\title{
DECOLONIZACIÓN EDUCATIVA FRENTE AL PENSAMIENTO EUROCÉNTRICO OCCIDENTAL
}

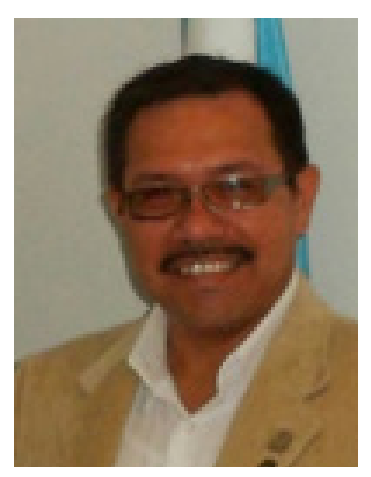

Fecha de recepción: 01/12/2017

\section{Pablo Gómez}

Universidad Nacional Experimental "Rafael María Baralt", Venezuela pabloramongomeznavarro@gmail.com

\section{RESUMEN}

Elpresenteensayoesunareflexiónsobreladecolonizacióneducativa frentealpensamientoeurocéntrico presente aun en nuestro escenario educativo actual. En él se analizan los diferentes discursos e ideas que esbozan los principales pensadores de la decolonización. En este sentido, se plantea estudiar desde una metodología documental, las ideas de autores tales como: Freire (2002), Santos (2010), Fernández (2011), Mejías (2010), Walsh (2014), Solano (2014), Fannon (2014), entre otros. Tales planteamientos tienen el propósito de entender las posiciones discursivas de estos autores orientados a romper las cadenas mentales que atan a la institución educativa formal a prácticas neocoloniales y proponer un nuevo modelo educativo liberador a partir de la visión emancipadora e historicista circunscrita en una pedagogía crítica de resistencia contra la ideología dominante del sistema opresor.

Palabras clave: decolonización, epistemología, eurocéntrico, pensamiento decolonial, emancipación.

\section{EDUCATIONAL DECOLONIZATION AGAINST THE WESTERN EURO-CENTRAL THOUGHT}

\begin{abstract}
The present essay is a reflection on educational decolonization against Eurocentric thought present even in our current educational scenario. In him the different speeches and ideas that outline
\end{abstract}


Decolonización educativa frente al pensamiento eurocéntrico occidental

Pablo Gómez (79:88)

the main thinkers of the decolonization are analyzed. In this sense, it is proposed to study from a documentary methodology, the ideas of authors such as: Freire (2002), Santos (2010), Fernández (2011), Mejías (2010), Walsh (2014), Solano (2014), Fannon (2014), among others. Such proposals have the purpose of understanding the discursive positions of these authors aimed at breaking the mental chains that bind the formal educational institution to neocolonial practices and proposing a new liberating educational model based on the emancipatory and historicist vision circumscribed in a critical pedagogy of resistance against the dominant ideology of the oppressive system.

Keywords: decolonization, epistemology, Eurocentric, decolonial thinking, emancipation.

* Profesor Titular adscrito al Departamento de Ciencias gerenciales, Doctor en Ciencias de la Educación, Ph.D en Gerencia de la Educación Superior, Especialista en Metodología de la Investigación, Participante de la Maestría en Filosofía mención: Pensamiento Latinoamericano.

\section{INTRODUCCIÓN}

En este mundo definido desde la visión capitalista como "globalizado", la educación actual, afronta una crisis caracterizada por las complejidades, relativismos éticos y contradicciones, donde el conocimiento fraccionado, especializado y demarcado por criterios de dominación que desalienta la comprensión integradora del ser, niega otras formas alternativas (no occidentales) de comprensión e interpretación de la realidad social y educativa.

Considerando lo antes expresado, este ensayo tiene como propósito reflexionar sobre la decolonización educativa frente al pensamiento eurocéntrico occidental a partir de las ideas de autores como Freire (2002), Solano (2015), Walsh (2014), Sousa (2010), Fernández (2011) Ducasse (2017), Fanon (2003), Dussel (2012), sobre la decolonización educativa como una alternativa destinada a subvertir las prácticas educativas coloniales, además de plantear la deconstrucción cultural y decolonización antropocéntrica a fin de visibilizar nuestra cultura e historia ancestral como pueblo en resistencia frente al pensamiento eurocéntrico presente aun en nuestros escenarios educativos actuales.

Lo anteriormente planteado, valida la tesis sobre la necesidad de repensar nuevas formar de organizar la educación para enfrentar no solo las complejidades de la sociedad actual, sino también romper las cadenas que nos atan al pensamiento eurocéntrico occidental a partir de una educación decolonizadora comprometida a valorar la identidad e historia de los pueblos latinoamericanos. 
Finalmente, el ensayo presenta una estructura dividida en tres partes: en primer lugar, una breve historia sobre el pensamiento eurocéntrico y la escuela formal latinoamericana; en segundo lugar, el pensamiento decolonial y la educación Latinoamérica actual y en tercer lugar, las nuevas formas de la educación desde una pedagogía crítica.

Una breve historia sobre el pensamiento eurocéntrico y la escuela formal latinoamericana.

Al tratar el pensamiento eurocéntrico en la escuela, se inicia rememorando nuestra historia latinoamericana marcada por la acción invasora colonial-imperial que llevaría a la imposición de una visión antropocéntrica cargada de valores y creencias la cual llevaría a invisibilizar la cosmovisión originaria de nuestros pueblos.

Esta acción llevada a cabo por los centros de poder coloniales de la época, traería consigo un proceso de alienación que deformó e instauró un pensamiento arraigado por el desprecio hacia otros pueblos(no europeos), lo que coincide con el punto de vista de Fanon (2003:35, 36), al señalar que "no bastó con reducir los espacios del colonizado, sino que el colonizador desfiguraría y deformaría también el imaginario del colonizado, afianzado su poder colonial y subyugándolo a una esclavitud mental".

En este sentido, América Latina tal y como lo expresa Solano (2014:118), experimentó uno de los más brutales procesos de colonización y subalternización que han vivido los seres humanos sobre la faz de la tierra. En cuestión de 50 años, los conquistadores no solo impusieron su propia visión de mundo, sino que desestructuraron y aniquilaron civilizaciones cuya riqueza material y espiritual aún no han sido sopesados en su totalidad.

La fuerte violencia racial marcada durante el proceso colonial, se orientaría a imponer una única visión del mundo generando nuevas instituciones lo que trajo como consecuencia la instauración de nuevas prácticas sociales que redefinieron la manera de pensar de colonizado y legitimando las acciones que llevarían a la destrucción su cultura originaria. Esto trajo como consecuencia lo expresa por Solano (2014:129), "una huella indeleble al erigirse una cultura como prototipo de sociedad ideal a seguir, generado la negación y, la mayoría de las veces, el rechazo e invisibilización de la otredad". 
Decolonización educativa frente al pensamiento eurocéntrico occidental

Pablo Gómez (79:88)

Ante esta realidad histórica colonial demarcada en un proceso de negación cultural llevaría a convertir la actual escuela y los procesos de educación formal en una institución reproductora de acciones alienantes encaminadas a reafirmar la idea de una sociedad civilizada sujeta a patrones de comportamiento euro-Occidental, lo que coincide con lo expresado por Cruz y et al (2012:14), "el discurso opresor hegemónico de la modernidad convierte la institución educativa en incapaz de asumir las diferencias de los sujetos y niega otros saberes en la medida en que margina al sujeto y su cultura".

La acción deliberada desde las estructuras de la educación formal está oculta en las ideas de progresismo que surgen en la década de los sesenta y donde la escuela sería el pilar fundamental del pensamiento occidental economicista, el cual solo deja ver el pensamiento originario ancestral como una muestra del atraso y barbarie hacia nuestros pueblos, denominándolos subdesarrollados.

Para Carreño (2010:196), dicha teoría del desarrollismo significó un proceso lineal que parte del subdesarrollo, después atraviesa varias etapas para llegar finalmente al desarrollo, situado al otro extremo final del proceso, y que le otorga a la educación un sector auxiliar para sus propósitos económicos. Toda vez que, como señala Carreño (2010), le adjudico la tarea de preparar recurso humano para lograr llegar a fin ese proceso lineal.

Lo anteriormente planteado, llevaría a romper con la visión desarrollista lineal y dominante desde una perspectiva crítica que busque a partir de la una ruptura epistemológica que busque a partir de la transformación del sistema educativo reproductor heredado cambio hacia nuevas formas de pensar la educación como emancipadora al ser capaz de subvertir las prácticas educativas colonizadoras como expresa Walsh (2017:498) al mencionar que "la decoloneidad del saber estaría relacionado a un cambios en la dirección de cómo razonamos y sentimos nuestra realidad social, lo que implicaría avanzar hacia nuevos proyectos sociales, culturales y políticos desaprendiéndonos de las ataduras de pasado colonial”. 


\section{El pensamiento decolonial y la educación en Latinoamérica actual.}

La sociedad latinoamericana, se ve enfrentada a diversos desafíos de adecuación. Esto implicaría, que frente a la realidad educativa actual marcada por una visión donde lo económico y tecnificado es la prioridad; y una educación latinoamericana que según Restrepo y Rojas (2010; 37-38), exhibe fuertes demarcaciones entre el saber académico, científico, filosófico y el saber cotidiano se requeriría romper con los principios y valores del modelo educativo servil del pensamiento colonial, para construir un nuevo proyecto educativo liberador y contra hegemónico sustentado en el respeto a las distintas cosmovisiones de los pueblos latinoamericanos.

Todo lo anterior, coincide con los planteamientos de Ducasse (2015:19) que busca reivindicar proyectos políticos liberadores opuestos a una educación de mercado, donde la cultura autoritaria reproduce acríticamente en la escuela e instituciones educativas el patrón de dominación.

Por tanto, la pedagógica critica supondría un viraje hacia una educación subversiva donde las contradicciones con el modelo dominante orientaría la formación de un ser humano capaz de interpreta su realidad histórica para comprender su posición en la sociedad llevando a reconstruir su contexto social y redefinir su sistema simbólicos que da cuerpo a las prácticas sociales en función de sus intereses colectivos más allá del estado omnipresente opresor.

En concordancia con lo manifestado por Solano (2015:123), un combate que debe conducir a la emancipación de las cadenas mentales que atan la educación, la escuela, la pedagogía, la reproducen el imperio de la razón y el conocimiento frío y vacío que acompaña a los procesos desubalternización sociocultural con los que se nos enseñó a comprender la realidad e insertarnos en ella. A parte, la formación educativa la cual como señala Fernández (2010), orientada hacia una perspectiva en la que lo educativo es profundamente político implica el reconocimiento de situaciones de injusticia, opresión, colonización y subalternidad de los sujetos para construir un proceso de concientización desde allí. De manera que, se establezca una pedagogía donde lo humano reconfiguren la acción educativa liberadora frente a los cambios que la sociedad latinoamericana viene experimentado.

Para América Latina y específicamente Venezuela, el desafío se orientaría a dar respuesta a las demandas sociales que han emergido en estos últimos años donde las inadecuaciones en el tipo de formación educativa, los procesos excluyentes, la poca vinculación de los programas con la realidad social, son elementos que llevan a repensar a la educación hacia nuevas formas 
Decolonización educativa frente al pensamiento eurocéntrico occidental

Pablo Gómez (79:88)

organizativas desde una perspectiva descolonizadora lo cual refuerza lo expresado por De Oto, A. (2010:285), confronta desde una práctica pedagógica al poder que domina y coloniza al sujeto, los saberes e historia.

Ante esta situación, la construcción de un nuevo pensamiento educativo emerge sobre las bases de la emancipación del sery la visión historicista que convierte un proceso complejoy de largo alcance comoposible, pues supone, des-aprenderparare-aprenderapartirdeunabaseteóricasustentada, bajoun cuerpopolítico-ideológicoaccionandodesdedonde,laprácticadedecolonizaciónbuscaríaimplosionar toda la base que sostiene el sistema educativo reproductor y el pensamiento eurocéntrico dominante.

\section{Las nuevas formas de la educación desde una pedagogía crítica.}

Ante el reduccionismo que según Mejías (2011:47), se plantea a la educación como mera formadora de estándares y competencias para una sociedad economicista, surge la pedagogía critica como práctica social nacida de las entrañas de los arreglos sociales y culturales latinoamericanos comprometida a transformar el sistema educativo formal a partir de una educación liberadora del ser. En concordancia con este planteamiento, autores como Mejías señala (2011:16), que hablar de educación y pedagogía crítica es hablar de una práctica social abierta que emerge de las propias realidades sociales, culturales y políticas de nuestra América Latina, enfrentándose a las injusticias sociales y transformando nuestras realidades.

De manera que, la estrategia se orienta a crear una visión contra hegemónica nacida de una visión liberadora de los pueblos bajo una acción que toma el pensamiento originario indígena para crear una episteme propia que responda a las singularidades de nuestros pueblos y no a proyectos neocoloniales. En ese mismo orden de ideas, Solano (2015), destaca que la educación decolonial se orienta a crear una nueva visión de mundo, más humana, más solidaria y más crítica.

Lo anterior, llevaría a comprender la realidad humana desde una dimensión compleja más allá del capital, dejando atrás la objetivación del ser y la formación educativa mercantilista para reorientase hacia una educación subversiva que se enfrenta a los viejos preceptos y crea nuevas utopías sobre lo que ha de ser una nueva sociedad que supere el modelo capitalista dominante.

En este orden de ideas, Walsh (2013:25), expresas "lo decolonial denotaría, entonces, un camino de lucha continuo en el cual se puede identificar, visibilizar y alentar 'lugares' de 
exterioridad y construcciones alter-(n)atibas".

Esto significaría, crear no solo un nuevo contrato social orientado hacia una alternativa educativa emancipadora, sino también llegar a la construcción de nuevas formas de educación tal y como señala Ducasse (2015:38), ’La pedagogía crítica reconocería la politicidad en toda práctica educativa y rechaza la neutralidad, objetividad, universalismo y tecnificación o sea se entrelaza con proyectos políticos económicos y culturales que se engarzan con proyectos históricos"

En este sentido, la educación liberadora debe dejar atrás la educación tecnocrática sustentada en la lógica del capital que cosifica, margina y excluye al ser bajo una perspectiva histórica colonial marcada por la globalización mercantil para reorientarse hacia la construcción de un sujeto el cual a partir de un dialogo cultural construye un nuevo conocimiento el cual redefinir su realidad histórica, social y política desde la toma de conciencia convirtiendo al sujeto en agente transformador de su mundo y constructor de nuevas instituciones.

Por último, para nuestra Latinoamérica, el desafío de desarrollar nuevas formas de la educación desde una pedagogía critica del sur como paradigma alternativo pasa por la acción necesaria de fortalecer la visión latinoamericana integradora llevándonos por los caminos de la emancipación del saber, el reconocimiento en la diversidad y el conocimiento que emerge de nuestros propios proceso de construcción del saber.

\section{Al final del camino, una reflexión}

Al final de este ensayo, se reconocen las ideas de diferentes autores sobre la decoloneidad educativa latinoamericana y su lucha ante un modelo educativo formal reproductor del pensamiento eurocéntrico y dirigido a responder a la perspectiva economicista de modelo capitalista dominante.

Dado los desafíos de la complejidad social los movimientos populares exigen que la educación sea capaz de emancipar a los individuos para romper las cadenas que atan a la visión eurocéntrica. Es así como, la decolonización educativa buscaría contribuir al debate crítico sobre nuevas alternativas educativas, reconociendo la responsabilidad social de la educación de avanzar hacia nuevos caminos de progreso a la creación de nuevas utopías que lleven a la construcción de un ser humano dotado de principios éticos, comprometido a la comprensión de problemas polifacéticos y solución de las dimensiones sociales. 
Decolonización educativa frente al pensamiento eurocéntrico occidental

Pablo Gómez (79:88)

Estas acciones llevarían a reivindican los ideales de paz, solidaridad, equidad, igualdad, libertad, respeto a las diversas formas de pensamiento, la defensa de los derechos humanos, la valoración de la democracia participativa y protagónica y respeto a la autodeterminación de nuestros pueblos Latinoamericanos.

Lo contrario significaría, reafirmar desde el pensamiento colonial de la superioridad de la cultura occidental frente a la "barbarie" que simbolizó para los colonizadores nuestra cultura originaria ancestral. En ese sentido, para Solano (2015:123) "la educación decolonizadora supondría un combate que debe conducir a la emancipación de las cadenas mentales que atan la educación, la escuela, la pedagogía y la cultura a un modelo civilizatorio en crisis".

Por tanto, la diversidad y las nuevas formas de organizar el conocimiento, el ritmo veloz de su producción, la pluralidad de la ciencia y la complejidad de los nuevos saberes ya no permite que la transmisión de conocimientos se haga como se venía haciendo tradicionalmente; por lo tanto, se hace necesario llevar a cabo cambios para lograr una enseñanza más integral, compartida y transdisciplinaria.

Del mismo modo, que la educación enfrente el reto de generar nuevas formas educativas sostenidas en la necesidad de crear estructuras organizativas que redefinan las estrategias destinadas a mejorar la calidad educativa desde una perspectiva posindustrial, a través de programas y modelos educativos destinados a reorientar el papel de la educación desde una perceptiva constructivista, critica, reflexiva, dialógica, participativa, autogestionaria y liberadora.

Todo lo anterior, implicaría la construcción de una epistemología del sur, que reafirmaría la idea de decoloneidad educativa creada como respuesta epistémica y política frente a un pensamiento marcado por la exclusión y el dominio económico donde la visión hegemónica de la modernidad construye una creencia sostenida en la negación de los otros no europeos. 


\section{REFERENCIAS BIBLIOGRÁFICAS}

Carreño M, (2010), Teoría y práctica de una educación liberadora: el pensamiento pedagógico de Paulo Freire. Secretariado de publicaciones universidad de Sevilla. Cuestiones Pedagógicas, 20, 2009/2010, pp. 195-214

Cruz Pérez Oscar, Germán Alejandro, García Lara, Pérez Jiménez, Carlos entre otros (2012) La escuela: discursos, prácticas, rupturas y tensiones. Editorial Unicach México

De Oto, A. (2010), "Pensamiento descolonial/decolonial”, en Proyecto: Diccionario del pensamiento alternativo II. Pensamiento latinoamericano y alternativo. Cecies. Disponible en: http://www.cecies.org/articulo.asp?id=285

De Souza Silva, José (2010) La pedagogía de la pregunta y el ‘día después del desarrollo’ Hacia la educación contextualizada para construir el buen vivir en el mundo rural latinoamericano1 Documento de apoyo a la presentación del autor, el día 8 de julio de 2010, en el "III Congreso Nacional de Educación Rural” realizado en Medellín, Colombia.

Dussel, E. (2012), Erótica y Pedagógica. Para una ética de la liberación Latinoamericana. Obras selectas VIII. Docencia, Buenos Aires.

Fabián Cabaluz Ducasse (2015) Entramando Pedagogías Críticas Latinoamericanas. Notas teóricas para potenciar el trabajo político-pedagógico comunitario. Editorial Quimantú Colección: A-probar Santiago de Chile

Fannon, Frank. (1963). Los condenados de la tierra. México: Editorial Fondo de Cultura Económica

Fernández Mouján, I. (2010), “En la educación: las marcas de la colonialidad y la liberación”, en Revista Sul-Americana de Filosofía e Educação. Número 15, nov/2010-abr/2011, pp. 5579. Disponible en: http://seer.bce.unb.br/index.php/resafe/issue/view/603.

Freire, P. (1970/2002). Pedagogía del Oprimido, Siglo XXI, Buenos Aires, pág. 72. 
Decolonización educativa frente al pensamiento eurocéntrico occidental

Pablo Gómez (79:88)

Mejías, Marcos (2011) Educaciones y pedagogías criticas del sur, ediciones vice ministerio de educación alternativa y especial Paz- Bolivia

Pedraza Flores, René (2014). Pedagogía práctica Del para la educativa siglo XXI Universidad Autónoma del Estado de México

Solano Alpízar, José (2015) Descolonizar la educación o el desafío de recorrer un camino diferente Revista Electrónica Educare (Educare ElectronicJournal) EISSN: 1409-4258 Vol. 19(1) ENERO-ABRIL, 2015: 117-129[Número publicado el 01 de enero del 2015]

Walsh, Catherine (2017) Pedagogías decoloniales: Prácticas insurgentes de resistir, (re)existir y (re)vivir. TOMO II Ediciones Abya- Yala Quito-Ecuador 